\title{
Fabrication and Efficiency Evaluation of a Hybrid NiCrAl Pressure cell up to $4 \mathrm{GPa}$
}

\section{$\operatorname{AUTHOR(S):~}$}

Fujiwara, Naoki; Matsumoto, Takehiko; Nakazawab, Kazuko-; Hisada, Akihiko; Uwatoko, Yoshiya

\section{CITATION:}

Fujiwara, Naoki ... [et al]. Fabrication and Efficiency Evaluation of a Hybrid NiCrAl Pressure cell up to 4 GPa. Review of Scientific Instruments 2007, 78(7): 073905.

\section{ISSUE DATE:}

2007-07

URL:

http://hdl.handle.net/2433/241770

\section{RIGHT:}

This article may be downloaded for personal use only. Any other use requires prior permission of the author and AIP Publishing. This article appeared in (citation of published article) and may be found at https://aip.scitation.org/doi/10.1063/1.2757129.; The full-text file will be made open to the public on 1 July 2008 in accordance with publisher's 'Terms and Conditions for Self-Archiving' 


\title{
Fabrication and efficiency evaluation of a hybrid NiCrAl pressure cell up to $4 \mathrm{GPa}$
}

\author{
Naoki Fujiwara ${ }^{a}$ \\ Graduate School of Human and Environmental Studies, Kyoto University, Nihonmatsu, Sakyo-Ku, Kyoto \\ 606-8501, Japan \\ Takehiko Matsumoto \\ National Institute for Material Science, 1-2-1 Sengen, Tsukuba, Ibaraki 305-0047, Japan
}

Kazuko Koyama-Nakazawa

ISSP, University of Tokyo, 5-1-5 Kashiwanoha, Kashiwa 277-8581, Japan

\begin{abstract}
Akihiko Hisada
Graduate School of Human and Environmental Studies, Kyoto University, Nihonmatsu, Sakyo-Ku, Kyoto 606-8501, Japan
\end{abstract}

Yoshiya Uwatoko

ISSP, University of Tokyo, 5-1-5 Kashiwanoha, Kashiwa 277-8581, Japan

(Received 19 January 2007; accepted 18 June 2007; published online 19 July 2007)

\begin{abstract}
A hybrid $\mathrm{NiCrAl}$ pressure cell was fabricated to measure magnetic quantities under high pressure above $3 \mathrm{GPa}$. A pressure of $4.0 \mathrm{GPa}$ was achieved and the pressure cell was found to be reusable even after a pressurizing trial up to $4.0 \mathrm{GPa}$. Pressure was monitored using ${ }^{63} \mathrm{Cu}$ nuclear quadrupole resonance of $\mathrm{Cu}_{2} \mathrm{O}$ and ruby fluorescence. The pressure efficiency of a fresh cell was maintained at $96 \%$, and no appreciable deformation was observed at pressures below $3 \mathrm{GPa}$; on the other hand, the efficiency after pressurizing trials decreased gradually and reached $75 \%$ at $4 \mathrm{GPa}$ accompanied by a maximum expansion inside the cylinder of 2\%. () 2007 American Institute of Physics.
\end{abstract}

[DOI: $10.1063 / 1.2757129]$

\section{INTRODUCTION}

Pressure has became increasingly important in the research on strongly correlated electron systems, because several $3 d$-transition-metal oxides, heavy fermion systems, and organic conducting systems exhibit a variety of phases with applied pressure. The phases include charge-ordering $(\mathrm{CO})$, magnetic ordering, paramagnetic metallic, and superconducting (SC) states. The variety originates from strong correlation or many-body effect of the electron systems. In the case of pressure-induced superconductivity, a spin -or chargeordering phase often appears at the lower pressure side on a pressure-temperature $(P-T)$ phase diagram. Coexistence of the ordering states with the SC state, or anomalous metallic behavior near the quantum critical point $(\mathrm{QCP})$, where the ordering state becomes unstable, is one of the current important topics in strongly correlated electron systems.

As examples of pressure-induced superconductors, the following are well-known compounds: spin-ladder cuprate, $\mathrm{Sr}_{14-x} \mathrm{Ca}_{x} \mathrm{Cu}_{24} \mathrm{O}_{41},{ }^{1,2} \beta$ bronzes, $A \mathrm{~V}_{6} \mathrm{O}_{15}(A=\mathrm{Li}, \mathrm{Na}$, and $\mathrm{Ag}$ ), ${ }^{3,4}$ Bechigaard salts such as (TMTSF) ${ }_{2} \mathrm{PF}_{6}$ (Refs. 5 and 6) and (TMTTF) ${ }_{2} \mathrm{PF}_{6},{ }^{7}$ and heavy fermion systems such as $\mathrm{CeCu}_{2} \mathrm{Ge}_{2}$ (Ref. 8) and $\mathrm{UGe}_{2}$. ${ }^{9}$ In the spin-ladder cuprate $\mathrm{Sr}_{14-x} \mathrm{Ca}_{x} \mathrm{Cu}_{24} \mathrm{O}_{41}$, measurements of dielectric constant and resistivity suggested the appearance of a charge-densitywave (CDW) at the Ca-poor region of $x \leqslant 10$ and the disap-

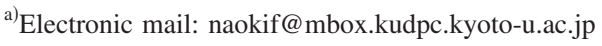

pearance at the Ca-rich region of $x \geqslant 10 .{ }^{10}$ Superconductivity has been observed at the Ca-rich region with application of pressure of over $3 \mathrm{GPa}^{1}$ Recently, superconductivity of $\beta$ bronzes has been discovered by applying pressures of over $6 \mathrm{GPa}^{4}$ In this series, the SC phase is located on the high pressure side of the $\mathrm{CO}$ phase. ${ }^{4}$ For (TMTSF) ${ }_{2} \mathrm{PF}_{6}$ and $(\mathrm{TMTTF})_{2} \mathrm{PF}_{6}$, the $\mathrm{SC}$ phase appears next to a spin-densitywave (SDW) phase on the $P-T$ phase diagram. The onset pressures where superconductivity emerges are 1 and $4 \mathrm{GPa}$ for the former and the latter, respectively., ${ }^{5,7} \mathrm{In} C \mathrm{com}$ pounds, the SC phase is located on the high pressure side of an antiferromagnetic (AF) phase on the $P$ - $T$ phase diagram, and coexistence with the SC state has been discussed as mentioned above.

Among these compounds, some have been precisely investigated using various techniques under pressure, whereas the others have not. It is an important factor whether the onset pressure is achieved by a piston-cylinder-type pressure cell. The current borderline lies around a pressure of 2.7-3.0 GPa.

The simplest pressure cell is made of $\mathrm{CuBe}$, whose upper limit is at most $1.5 \mathrm{GPa}$. The upper limit of a Ti-alloy pressure cell is at most $2 \mathrm{GPa}^{11,12}$ The upper limit of a hybrid pressure cell with an outer $\mathrm{CuBe}$ sleeve and inner $\mathrm{WC}$ is at most $3 \mathrm{GPa} .{ }^{13}$ Recently, NiCrAl or MP35N cylinders have attracted much attention as post-WC cylinders, because they are nonmagnetic and possess high tensile and yield strengths. ${ }^{14-16}$ In paticular, NiCrAl contains fewer impurities 


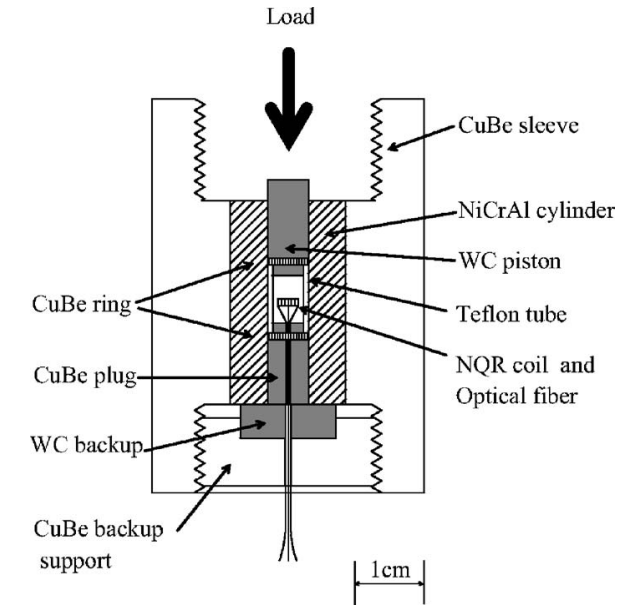

FIG. 1. Scheme of a NiCrAl pressure cell used in the present experiment. $\mathrm{CuBe}$ rings are set on the $\mathrm{WC}$ piston and the $\mathrm{CuBe}$ plug to prevent a leak of pressure meditation liquid. A NQR coil is wrapped around powder samples of $\mathrm{Cu}_{2} \mathrm{O}$ and sintered into the liquid. An optical fiber and electric leads go through the pressure cell. Inside of the CuBe plug is sealed with Stycast epoxy resin. Length of each component is shown in the text.

than MP35N and is suitable for magnetic measurements. ${ }^{15}$ In fact, microscopic investigation of superconductivity in $\mathrm{Sr}_{14-x} \mathrm{Ca}_{x} \mathrm{Cu}_{24} \mathrm{O}_{41}$ became possible by the development of a NiCrAl pressure cell. ${ }^{17}$ The cuprate spin-ladder system is a representative that invokes the significance of the $\mathrm{NiCrAl}$ pressure cell. To promote researches on a wide variety of materials, the development and precise characterization of the nonmagnetic pressure cell are of great importance. However, the upper limit of the NiCrAl pressure cell is still an open problem, because it strongly depends on the design of the pressure cell as well as the strengths of the material.

In the present work, we applied loads up to 15.0 tons to a hybrid NiCrAl pressure cell and investigated the pressure limit and the efficiency.

\section{PRESSURIZING APPARATUS}

\section{A. Pressure cell}

Figure 1 shows a scheme of a hybrid pressure cell fabricated in the present experiment. The length of the NiCrAl cylinder is $30 \mathrm{~mm}$, and the inner and outer diameters are 6 and $16 \mathrm{~mm}$, respectively. The cylinder is covered by a $\mathrm{CuBe}$ sleeve with an outer diameter of $40 \mathrm{~mm}$. The outside of the cylinder and the inside of the sleeve are tapered off, and the cylinder is inserted into the sleeve by a force from a press. A mixture of fluorinated liquids, Fluorinert FC-70 and FC-77, was prepared as a pressure mediation liquid. This mixture remains hydrostatic up to $10 \mathrm{GPa}$. A Teflon tube with an inner volume of $4.4 \mathrm{~mm}$ diameter $\times 15 \mathrm{~mm}$ length was filled with the Fluorinert mixture. Both ends were closed by a WC piston and a $\mathrm{CuBe}$ plug. The inner liquid was sealed with $\mathrm{CuBe}$ rings. An optical fiber and an nuclear quadrupole resonance (NQR) coil were equipped together in the sample space. We used a fiber of $0.25 \mathrm{~mm}$ outer diameter and a coil of $2 \mathrm{~mm}$ length with a winding of 25 turns. Ruby powder was fixed using a quick-drying glue on the top of the fiber. The position of the ruby powder was $2 \mathrm{~mm}$ above the top of the $\mathrm{CuBe}$ plug.

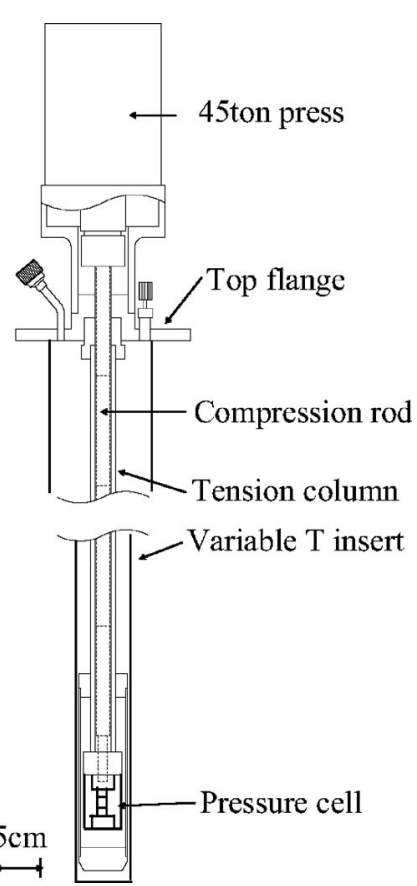

FIG. 2. Steady-load control system equipped on the top flange of variabletemperature insert (VTI). A load is transferred to the pressure cell via a compression rod. The pressure cell was screwed onto a tension column. The load is supported by the tension column, the CuBe sleeve, and backup support. These components act as a frame of a conventional press system. ${ }^{63} \mathrm{Cu} \mathrm{NQR}$ of $\mathrm{Cu}_{2} \mathrm{O}$ was performed using this equipment. Length of each component is shown in the text.

\section{B. Load transmitting system}

Two types of press systems are prepared to apply the load: one is a conventional system and the other is a special version arranged on the top flange of variable-temperature insert (VTI) (Fig. 2). The length between the top flange and the bottom of the VTI is $1340 \mathrm{~mm}$. The pressure cell is screwed into the tension column made of MP35N, and the load is transferred to the pressure cell mediated by the inner compression rod. The tension column, the CuBe sleeve, and the $\mathrm{CuBe}$ backup support act as a frame in a conventional press system. Steady-load control becomes possible owning to feedback from the pressure cell, even at low temperatures where pressure decrease is expected because of contraction of pressure mediation liquid. The apparatus is similar to the type designed by Oomi et al. ${ }^{18}$ for resistivity measurements. In the present VTI, two trimmer capacitors are equipped near the pressure cell to adjust NQR frequency and impedance matching. They are connected to tuning knobs outside the top flange by extension rods and can be controlled even at low temperatures.

\section{PRESSURE EVALUATION}

${ }^{63} \mathrm{Cu}\left(I=\frac{3}{2}\right)-\mathrm{NQR}$ measurement of $\mathrm{Cu}_{2} \mathrm{O}$ and ruby fluorescence are very useful as means of pressure calibration because they do not require any sweeps of physical parameters. One of the popular calibration methods at room temperatures is utilizing the structural phase transition of $\mathrm{Bi}$ or Te; the other at low temperatures is utilizing the supercon- 
ducting phase transition of $\mathrm{Pb}$ or $\mathrm{Sn}$. However, the former and the latter require pressure and temperature sweeps in the resistivity measurements, respectively.

\section{A. NQR}

$\mathrm{Cu}_{2} \mathrm{O}$ is often used for pressure calibration in nuclear magnetic resonance (NMR) because the NQR frequency corresponding to $\left|\frac{3}{2}, \pm \frac{3}{2}\right\rangle \Leftrightarrow\left|\frac{3}{2}, \pm \frac{1}{2}\right\rangle$ transition shows a linear relation with applied pressure. The NQR-frequency measurement below $2 \mathrm{GPa}$ has been performed at temperatures from 4 to $300 \mathrm{~K}$. ${ }^{19}$ The NQR frequency of $\mathrm{Cu}_{2} \mathrm{O}$ at room temperatures is estimated to be

$$
\nu(\mathrm{MHz})=26.0+0.33 P(\mathrm{GPa}),
$$

at pressures below $2 \mathrm{GPa} .{ }^{19,20}$ The NQR frequency is proportional to the electric field gradient (EFG) at a ${ }^{63} \mathrm{Cu}$ site. If the EFG is determined by the surrounding ions, it is proportional to the inverse of the volume. The linear relation implies that the compressibility of $\mathrm{Cu}_{2} \mathrm{O}$ is pressure independent.

\section{B. Ruby fluorescence}

Ruby fluorescence is often adopted for diamond anvil cells. The shift of the R1 transition also shows a linear relation with pressure. Ruby fluorescence has been confirmed to be a reliable calibration method, and pressure-calibration curves have been presented in several studies. ${ }^{21-23}$ The pressure range we measured is rather close to that performed by Piermarni et al. $^{21}$ They measured pressure up to $19.5 \mathrm{GPa}$ and obtained the fitting curve as

$$
P(\mathrm{GPa})=\Delta \lambda / 0.365(\mathrm{~nm}) \text {. }
$$

In a conventional method using a diamond anvil cell, only a piece of ruby is inserted in the cell. In our measurements, the ruby fixed on the top of the optical fiber was inserted into the sample space.

\section{EXPERIMENTAL RESULTS}

We performed the ${ }^{63} \mathrm{Cu}$ NQR of $\mathrm{Cu}_{2} \mathrm{O}$ and ruby fluorescence simultaneously using the steady-load control system mentioned above. The apparatus also avoids the repetition process, which is needed when the conventional press system is used, i.e., removing and attaching the pressure cell to a NQR probe. We used the conventional press only for ruby fluorescence.

We performed the following three pressurizing trials at room temperature.

(1) First trial with the conventional press up to 10.7 tons. In this trial we only measured the R1 shift using a fresh pressure cell.

(2) Trial with the VTI up to 15.0 tons. We measured both the NQR frequency and R1 shifts using the same cell after the first trial.

(3) Second trial with the conventional press up to 15.0 tons. In this trial, we only measured the R1 shift using the same cell after the trial with the VTI.

The R1 line moved 1.17, 1.40, and $1.48 \mathrm{~nm}$ at the maximum load for the three trials, respectively. Using Eq. (2), the

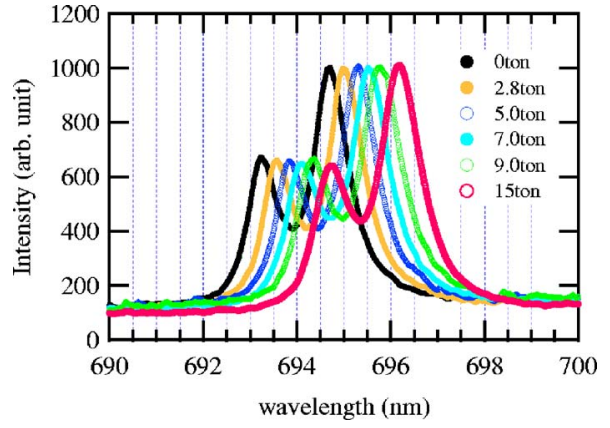

FIG. 3. (Color online) Typical spectra of ruby fluorescence measured with a conventional press up to 15.0 tons which corresponds to $4.0 \mathrm{GPa}$. The smaller and larger peaks correspond to the R2 and R1 transitions, respectively. Relation between an applied load and pressure is shown as open circles in Fig. 4.

pressures are estimated to be $3.2,3.8$, and 4.0 GPa for the three trials, respectively. Even at $4.0 \mathrm{GPa}$, no fatal damage was observed and the pressure cell was found to be reusable. Typical spectra up to 15.0 tons are shown in Fig. 3. The data were obtained during the pressurizing process up to 15.0 tons with the conventional press. In these spectra, broadening of the linewidth was hardly observed within the experimental accuracy. This is because the ruby powder occupies only a point in the sample space, and therefore, perfect homogeneity is not always realized in the whole sample space. Measurement at different ruby positions is needed to investigate the precise homogeneity in the sample space. Load dependence of the wavelength is shown in Fig. 4. In the first trial with a fresh cell, $\Delta \lambda$ shows almost a linear response, whereas in the other trials with the same cell, the linearity is limited at less than 5 tons. As seen from the figure, the curves tend to bend downward with increasing load. The trend becomes remarkable when the heavy load is imposed. The bend is attributable to the loss of efficiency during the pressurizing process.

Next, we show in Fig. 5 the load dependence of the NQR frequency measured with the VTI. We used a conventional coherent-pulsed NQR method. The NQR signal of $\mathrm{Cu}_{2} \mathrm{O}$ was observed as a free induction decay after a pulse of $5 \mu$ s. The data were obtained after an accumulation of 512 times. The accuracy of the frequency was within $\pm 0.02 \mathrm{MHz}$. In this trial a maximum pressure of $3.8 \mathrm{GPa}$ was achieved; however, the $\mathrm{Cu}_{2} \mathrm{O}$ signal was followed up to

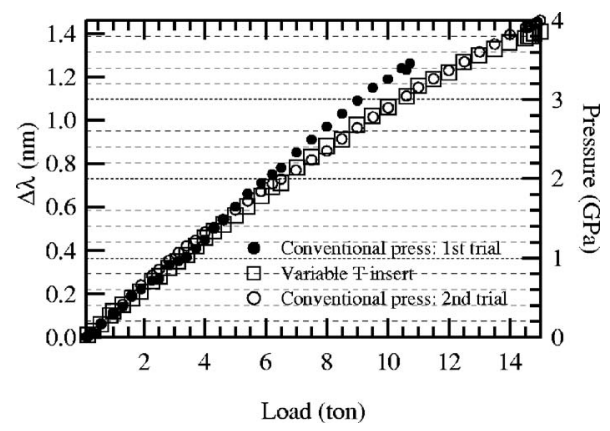

FIG. 4. Load dependence of the R1 shift in ruby fluorescence. The right axis is obtained from Eq. (2). Closed circles, open squares, and open circles represent the three trials explained in the beginning of Sec. III. 


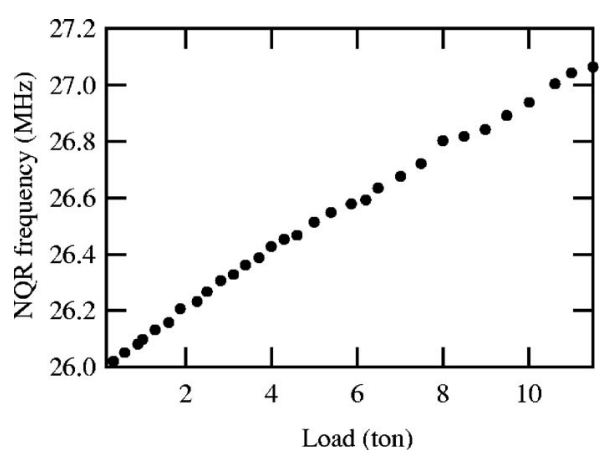

FIG. 5. Load dependence of the NQR frequency of $\mathrm{Cu}_{2} \mathrm{O}$ measured with the VTI shown in Fig. 2.

3.3 GPa. The signal became smaller when the heavy load was imposed. This may be attributed to the initial setup of impedance matching not being perfect. The signal also shifts from $26.0 \mathrm{MHz}$ linearly with a slope of $0.93 \mathrm{MHz} /$ ton at a light load. However, the linearity also breaks down at a heavy load as is the case of $\Delta \lambda$. The relationship between the NQR frequency and R1 shift is shown in Fig. 6. The linearity between the two quantities is good and is expressed as

$$
\nu(\mathrm{MHz})=26.0+0.883 \Delta \lambda(\mathrm{nm}) .
$$

From Eqs. (2) and (3), we obtained the pressure dependence of $\nu(\mathrm{MHz})$ as

$$
\nu(\mathrm{MHz})=26.0+0.32 P(\mathrm{GPa}) .
$$

The relation obtained from our results is consistent with that obtained below $2 \mathrm{GPa}^{19,20}$

\section{DISCUSSION}

The pressure efficiency is defined from the R1 shifts as

$$
\operatorname{Efficiency}(\%) \equiv \frac{\Delta \lambda(\mathrm{nm})}{0.365} \frac{S\left(\mathrm{~cm}^{2}\right)}{w(\text { ton })} \times 100 .
$$

We estimated the efficiency for the three trials, as shown in Figs. 7 and 8. Figure 8 is an expansion of Fig. 7 around the high efficiency region. In the first trial of the fresh cell, the efficiency reached $96 \%$ at 5.5 tons, and the value remained constant up to 10 tons. On the other hand, the efficiency of the other trials showed almost the same behavior at heavy loads. The efficiency of both trials decreased gradually from $90 \%$ to $75 \%$ above 4 tons. The loss of efficiency mainly arises from the deformation of the cylinder and the friction

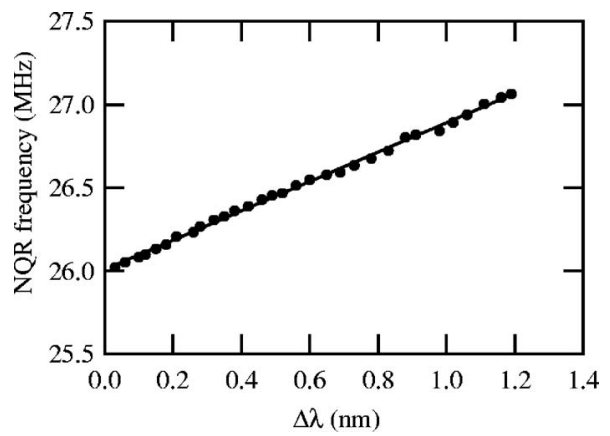

FIG. 6. Relation between the NQR frequency of $\mathrm{Cu}_{2} \mathrm{O}$ and the R1 shift which is shown in Fig. 4 as open squares. The solid line represents Eq. (3).

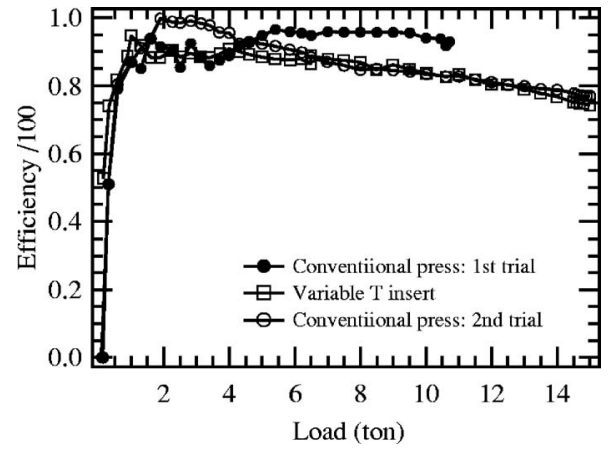

FIG. 7. Load dependence of efficiency defined by Eq. (5). Closed circles, open squares, and open circles represent the three trials explained in the beginning of Sec. III.

of the $\mathrm{CuBe}$ seal ring with the inner cylinder surface.

As for the deformation of the cylinder during pressurizing process, no appreciable expansion was observed for the first trial up to 10.7 tons. The load can be safely applied to the pressure cell at least up to $3.2 \mathrm{GPa}$. On the other hand, the inner diameter partly expanded over $2 \%$ when a load of 15.0 tons was applied. The detailed distribution of the expansion is shown in Fig. 9 with a scheme of the pressure cell, where the position and scale of each component are adjusted to the following figure. The dashed and solid lines represent the initial and final positions of the pressurizing process up to 15.0 tons, respectively. The maximum expansion is roughly located at the center of the sample space. As seen in Fig. 9, the expansion for the VTI is smaller than that for the conventional press. The expansion has a relation with the applied pressure rather than the applied load. The expansion is inevitable at the extreme condition.

The expansion certainly has a correlation with the applied pressure; however, the situation is much more complicated if one compares the present cell with other cells with different dimensions. We prepared another $\mathrm{NiCrAl}$ pressure cell of the same size except for the sleeve. We prepared a smaller sleeve with an outer diameter of $32 \mathrm{~mm}$. The results are shown in Fig. 10 with those for $40 \mathrm{~mm}$ measured with the conventional press (see pressurizing trial 2 in Sec. III). As seen from the figure, the efficiency fell to $77 \%$ even at 11.5 tons, corresponding to $3.0 \mathrm{GPa}$. In this case, the maximum expansion of the inner diameter reached $1 \%$, although no appreciable expansion was observed for the $40 \mathrm{~mm}$ sleeve. The expansion is not uniquely determined by the

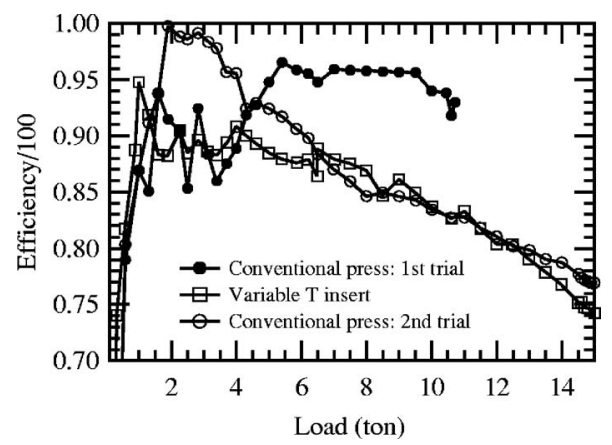

FIG. 8. Expansion of Fig. 7 at high efficiency region. The data are the same with those of Fig. 7. 

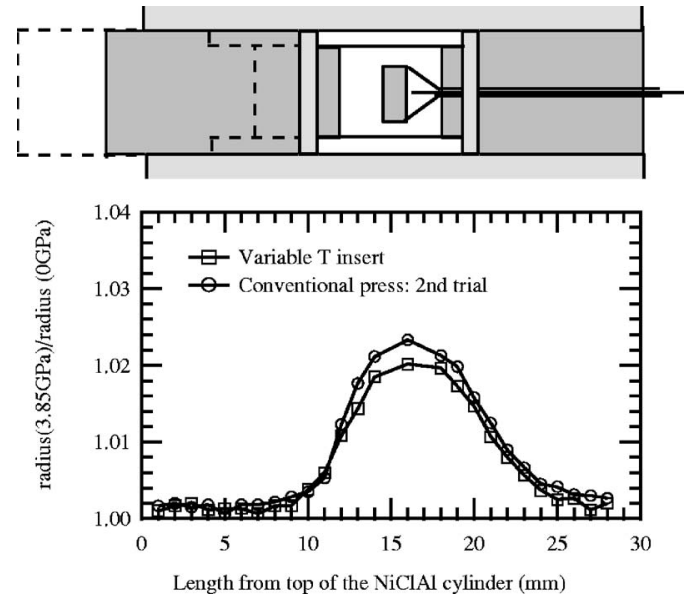

FIG. 9. Upper scheme represents the NiCrAl pressure cell at a load of 15.0 tons. Dashed lines represent the initial positions of the piston and the Teflon tube. The position and scale of the upper scheme are adjusted to the following figure which represents the expansion of inner diameter of the NiCrAl cylinder.

shape of the cylinder and applied pressure. The balance between the cylinder and the sleeve is an important factor in avoiding deformation of the cylinder.

The fracture strain of high-strength materials is usually not great, sometimes less than 3\%, therefore, the upper pressure limit of the NiCrAl cylinder is at most $4 \mathrm{GPa}$. Furthermore, a crack was observed in the WC piston after releasing the pressure from $4 \mathrm{GPa}$. The compression strengths are 6 and $4.5 \mathrm{GPa}$ for magnetic and the nonmagnetic WCs, respectively. In the present experiment, we used the nonmagnetic $\mathrm{WC}$, and therefore the appearance of the crack is not a surprise. To apply further pressure with this pressure cell, improvements to materials, especially for the piston, are also required as well as an effective sealing technique and the total design of the pressure cell.

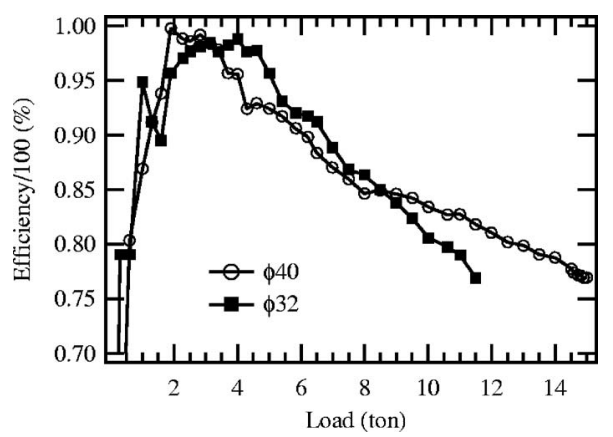

FIG. 10. Comparison of efficiency between sleeves with outer diameters of 32 and $40 \mathrm{~mm}$. The data for $40 \mathrm{~mm}$ are already shown in Figs. 7 and 8 as open squares.
We succeeded in applying pressures up to $4.0 \mathrm{GPa}$ using a nonmagnetic hybrid cell with a NiCrAl cylinder of $16 \mathrm{~mm}$ outer diameter and a CuBe sleeve of $40 \mathrm{~mm}$ outer diameter. The pressure cell could be reused even after applying a pressure of $4.0 \mathrm{GPa}$. The pressure efficiency of a fresh cell was maintained at $96 \%$, and no appreciable deformation was observed at pressures below $3.2 \mathrm{GPa}$; on the other hand, the efficiency after pressurizing trials decreased and reached $75 \%$ at $4 \mathrm{GPa}$ accompanied by a maximum expansion of the cylinder of $2 \%$.

\section{ACKNOWLEDGMENTS}

The authors wish to thank D. Teshima and T. Takahashi for experimental supports. This work was partially supported by a Grant-in-Aid for the Ministry of Education, Culture, Sports, Science and Technology, Japan.

${ }^{1}$ M. Uehara, T. Nagata, J. Akimitsu, H. Takahashi, N. Mori, and K. Kinoshita, J. Phys. Soc. Jpn. 65, 2764 (1996).

${ }^{2}$ T. Nagata et al., Phys. Rev. Lett. 81, 1091. (1998).

${ }^{3}$ Y. Ueda, J. Phys. Soc. Jpn. 69, 149. (2000).

${ }^{4}$ T. Yamauchi, Y. Ueda, and N. Môri, Phys. Rev. Lett. 89, 057002. (2002).

${ }^{5}$ D. Jerome, A. Mazaud, M. Ribault, and K. Bechgaard, J. Phys. Lett. 41, L195 (1980).

${ }^{6}$ I. J. Lee, S. E. Brown, W. G. Clark, M. J. Strouse, M. J. Naughton, W. Kang, and P. M. Chaikin, Phys. Rev. Lett. 88, 017004 (2001).

${ }^{7}$ D. Jaccard, H. Wilhelm, D. Jerome, J. Moser, C. Careel, and J. M. Fabre, J. Phys.: Condens. Matter 13, L89 (2001).

${ }^{8}$ D. Jaccard, H. Wilhelm, K. Alami-Yadri, and E. Vargoz, Physica B 259261, 1 (1999).

${ }^{9}$ S. Saxena et al., Nature (London) 406, 587 (2000).

${ }^{10}$ T. Vuletić et al., Phys. Rev. Lett. 90, 257002 (2003).

${ }^{11}$ I. R. Walker, Cryogenics 45, 87 (2005).

${ }^{12}$ K. Kamishima, M. Hagiwara, and H. Yoshida, Rev. Sci. Instrum. 72, 1472 (2001).

${ }^{13}$ H. Fujiwara, H. Kadomatsu, and K. Tohma, Rev. Sci. Instrum. 51, 1345 (1980).

${ }^{14}$ M. I. Eremets, High Pressure Experimental Methods (Oxford, New York, 1996).

${ }^{15}$ Y. Uwatoko, S. Todo, K. Ueda, A. Uchida, M. Kosaka, N. Mori, and T. Matsumoto, J. Phys.: Condens. Matter 14, 11291 (20021).

${ }^{16}$ I. R. Walker, Rev. Sci. Instrum. 70, 3402 (1999).

${ }^{17}$ N. Fujiwara, N. Môri, Y. Uwatoko, T. Matsumoto, N. Motoyama, and S. Uchida, Phys. Rev. Lett. 90, 137001 (2003).

${ }^{18}$ G. Oomi, T. Kagayama, and Y. Uwatoko, J. Appl. Phys. 32, 349. (1993).

${ }^{19}$ A. P. Reyes, E. T. Ahrens, R. H. Heffner, P. C. Hammel, and J. D. Thompson, Rev. Sci. Instrum. 63, 3120 (1992).

${ }^{20}$ T. Kushida, G. B. Beneddek, and N. Bloembergen, Phys. Rev. 104, 1364 (1956).

${ }^{21}$ G. J. Piermarni, S. Block, J. D. Barnett, and R. A. Forman, J. Appl. Phys. 46, 2774 (1975).

${ }^{22}$ H. K. Mao, P. M. Bell, J. W. Shaner, and D. J. Steinberg, J. Appl. Phys. 49, 3276 (1978).

${ }^{23}$ R. J. Hemley, C. S. Zha, A. P. Jephcoat, H. K. Mao, L. W. Finger, and D. E. Cox, Phys. Rev. B 39, 11820 (1989). 